TRANSACTIONS OF THE

AMERICAN MATHEMATICAL SOCIETY

Volume 357, Number 2, Pages 425-442

S 0002-9947(04)03627-X

Article electronically published on September 23, 2004

\title{
ON THE COMPLEXITY OF THE INTEGRAL CLOSURE
}

\author{
BERND ULRICH AND WOLMER V. VASCONCELOS
}

Dedicated to Aron Simis on the occasion of his sixtieth birthday

\begin{abstract}
The computation of the integral closure of an affine ring has been the focus of several modern algorithms. We will treat here one related problem: the number of generators the integral closure of an affine ring may require. This number, and the degrees of the generators in the graded case, are major measures of cost of the computation. We prove several polynomial type bounds for various kinds of algebras, and establish in characteristic zero an exponential type bound for homogeneous algebras with a small singular locus.
\end{abstract}

\section{INTRODUCTION}

Let $k$ be a field and let $A$ be a reduced ring which is a finitely generated $k-$ algebra. The integral closure $\bar{A}$ is also an affine algebra over $k$,

$$
A=k\left[x_{1}, \ldots, x_{n}\right] / I \hookrightarrow \bar{A}=k\left[y_{1}, \ldots, y_{m}\right] / J .
$$

The least $n$, among all such presentations of $A$, is the embedding dimension of $A$, $\operatorname{embdim}(A)$. We focus on the number of indeterminates needed to present $\bar{A}$. If $A$ is $\mathbb{N}$-graded, $\bar{A}$ is similarly graded and there will be presentations where the $y_{i}$ are homogeneous elements. We will denote by $\operatorname{embdeg}(\bar{A})$ the smallest value of $\max \left\{\operatorname{deg}\left(y_{i}\right)\right\}$ achieved among all presentations; we call that integer the embedding degree of $\bar{A}$.

These numbers are major measures for the complexity of computing $\bar{A}$. Our aim here is to give estimates for the embedding dimension of $\bar{A}$, and in the graded case for the embedding degree, under some restrictions. Whenever possible we will seek to control the embedding dimension of all intermediate rings which occur in the construction of $\bar{A}$. A major concern is to make use of known properties of $A$, such as information about its singular locus, or expected geometric properties of $\bar{A}$, in particular regarding its depth.

We introduce here a geometric approach to this problem. A direct approach would be to attempt to bound degree data on the presentation ideal $J$ in terms of $n$ and $I$. We will follow a different path, as we will assume that we possess information about $A$ with a geometric content, such as the dimension of $A$ and its multiplicity, and in certain cases some fine data on its singular locus. Our results

Received by the editors May 10, 2002.

2000 Mathematics Subject Classification. Primary 13B22; Secondary 13C15, 13H15, 13P10.

Key words and phrases. Cohen-Macaulay ring, integral closure, isolated singularity, Jacobian ideal, multiplicity.

The authors were partially supported by the NSF. 
will then be expressed by bounding either $\operatorname{embdim}(\bar{A})$ or embdeg $(\bar{A})$ in terms of these data.

In order to explain our results, let us bring out the invariants of an affine ring $A$ which are likely to occur in any estimate for embdim $(\bar{A})$ and embdeg $(\bar{A})$. We shall assume that $A$ is reduced and equidimensional of dimension $d$. One source of difficulty lies in the fact that the ring $B=\bar{A}$ may also be the integral closure of other rings. This fuzziness is at the same time a path to dealing with this problem in some cases of interest. After a possible change of variables, the subring generated by the images of the first $d$ variables $x_{i}$ is a Noether normalization of $A$,

$$
T=k\left[x_{1}, \ldots, x_{d}\right] \hookrightarrow A .
$$

We define the multiplicity $\operatorname{deg}(A)$ of $A$ to be the least rank of $A$ as a $T$-module for all possible Noether normalizations; this number is equal to the ordinary multiplicity $\operatorname{deg}(A)$ when $A$ is a standard graded $k$-algebra and $k$ is infinite. If $k$ is infinite and perfect, there exists an element $u \in A$ so that $\operatorname{deg}(A)=\operatorname{deg}(T[u])$. Notice that $T[u]=T[t] /(f)$ for some monic polynomial $f$ in $T[t]$ with $\operatorname{deg} f=\operatorname{deg}(A)$. Hence $S=T[t] /(f)$ is a subring of $A$ such that $B$ is also the integral closure of $S$. This shows that the only general numerical invariants we can really use for $\bar{A}$ are the dimension and the multiplicity of $A$, and to a lesser extent the Jacobian ideal of $S$.

There are many instances when an estimate for embdim $(\bar{A})$ is easy to obtain, for example the case where $\bar{A}$ is Cohen-Macaulay. The $\operatorname{ring} \bar{A}$ is then a free module over $T$, of $\operatorname{rank} \operatorname{deg}(A)$ having $T$ as a summand. Therefore $\operatorname{embdim}(\bar{A}) \leq \operatorname{dim} A+$ $\operatorname{deg}(A)-1$. For example, if $\operatorname{dim} A \leq 2$, this will always hold. Another instance are the rings $A$ generated by monomials; by the well-known theorem of Hochster ([13]), $\bar{A}$ will be Cohen-Macaulay.

Our overall aim is to derive elementary functions $\beta(d, e)$ and $\delta(d, e)$, polynomial in $e$ for fixed $d$, such that for any standard graded equidimensional reduced algebra $A$ of dimension $d=\operatorname{dim} A$ and multiplicity $e=\operatorname{deg}(A)$,

$$
\begin{aligned}
\operatorname{embdim}(\bar{A}) & \leq \beta(\operatorname{dim} A, \operatorname{deg}(A)), \\
\operatorname{embdeg}(\bar{A}) & \leq \delta(\operatorname{dim} A, \operatorname{deg}(A)) .
\end{aligned}
$$

The existence of non-elementary bounds, albeit not in any explicit form and therefore without the link to complexity, has been established in [7] 3.1] in a model theoretic formulation grounded on the explicit construction of integral closures of 22] and 25]. Our own estimates, tracking the constructions of $\bar{A}$ using the Jacobian methods of either [15] or [26], yield Gröbner bases bounds of very high order indeed: $\beta(d, e)$ comes in as a very "tall" function, with the number of levels of exponentiation quadratic in $e$. The bounds given here, beyond their effective character, seek to derive formulas for $\beta(d, e)$ and $\delta(d, e)$ that are sensitive to additional information known about $A$, such as the dimension of $A$ being at most 3 or the singular locus being small. To exercise this kind of control one must however work in restricted characteristics, usually zero.

We shall now outline the organization of the paper. We assume for simplicity that $A$ is a reduced and equidimensional algebra over a perfect field and that $A$ has dimension $d$ and multiplicity $e \geq 3$. In the next section we give the expected bound for embdim $(\bar{A})$ and, in the graded case, for embdeg $(\bar{A})$ provided $\bar{A}$ is CohenMacaulay. They will be $e+d-1$ and $e-2$ respectively (Theorem 2.1). In Section 3 we deal with graded rings of dimension 3 , or more generally with standard graded algebras $A$ over a field of characteristic zero for which $\operatorname{depth}_{A} \bar{A} \geq \operatorname{dim} A-1$. 
The bound obtained for embdim $(\bar{A})$ is $e(e-2)+d$ (Theorem 3.2 and Remark 3.3). In Section 4, we consider ways to deal with non-homogeneous algebras. It becomes harder to track multiplicities. Nevertheless, we obtain, under similar depth conditions as above, a general bound for $\operatorname{embdim}(\bar{A})$ (Theorem 4.3).

Heretofore we have used no numerical information on the algebra $A$ besides its dimension and multiplicity. In Section 5 we assume that $d \geq 4$ and that the singular locus of $A$ is sufficiently small. Roughly it is shown that if $A$ is standard graded over a field of characteristic zero and $A$ satisfies Serre's condition $R_{d-3}$, then

$$
\operatorname{embdim}(\bar{A}) \leq 1 / 2(e(e-1))^{2^{d-3}}-1 / 2(e(e-1))^{2^{d-4}}+2 d-2
$$

(Corollary 5.7; see also Theorem 5.6 for a more general estimate).

Section 6 deals with bounds on the embedding degree; they are restricted to standard graded algebras satisfying the condition $R_{1}$ at least, but are surprisingly low, as at worst we have found quadratic bounds. The first result (Theorem 6.3) has a conjectural aspect: If $A$ has characteristic zero and satisfies the condition $R_{2}$, then

$$
\operatorname{embdeg}(\bar{A}) \leq e(e-2)-1,
$$

provided $d-1$ is in the range where the Eisenbud-Goto conjecture holds ([8]). The other result (Theorem 6.5) is very pleasing: If $A$ satisfies $R_{1}$ and $\operatorname{depth}_{A} \bar{A} \geq d-1$, then

$$
\operatorname{embdeg}(\bar{A}) \leq 3 e-5 .
$$

In characteristic zero we are able to improve and extend this estimate using a result of Mumford (Theorem 6.6): If $A$ satisfies $R_{d-t}$ for $t=\operatorname{depth}_{A} \bar{A}$, then

$$
\operatorname{embdeg}(\bar{A}) \leq(d-t+1)(e-2) .
$$

We conclude with a comparative study of the bounds which the general complexity theory of Gröbner bases would provide under similar hypotheses. They will be consistently of a higher order of complexity.

\section{Cohen-Macaulay integral Closure}

We begin by introducing some notation and terminology and make some general comments. Throughout we use standard terminology: an affine $k$-algebra $A$ will denote a finitely generated algebra over a field $k$, and a standard graded $k$-algebra will be an $\mathbb{N}$-graded affine $k$-algebra generated by its homogeneous elements of degree 1. For general results in commutative algebra, particularly on Cohen-Macaulay rings, we refer the reader to 6 .

Suppose $A$ is an affine algebra, and $T=k\left[x_{1}, \ldots, x_{d}\right]$ is one of its Noether normalizations. The rank of $A$ over $T$ is the dimension of the $K$-vector space $A \otimes_{T} K$, where $K$ is the field of fractions of $T$. This dimension may vary with the choice of the Noether normalization. When $A$ is a standard graded algebra and the $x_{i}$ are homogeneous of degree 1 , this rank is equal to the multiplicity $\operatorname{deg}(A)$ of $A$ as provided by its Hilbert function. It is thus independent of the choice of such Noether normalization. In the non-graded case, by abuse of terminology, we let $\operatorname{deg}(A)$ denote the infimum of the ranks of $A$ over its various Noether normalizations, a terminology that is consistent in the case of standard graded algebras over infinite fields (see also [24, p. 251]).

If $S$ is a ring, we write $\nu_{S}(\cdot)$ for the minimal number of generators of $S$-modules. 
Theorem 2.1. Let $A$ be a reduced and equidimensional affine algebra over a field $k$ with $e=\operatorname{deg}(A)$. Let $A \subset B$ be a finite and birational ring extension, and assume that $B$ is Cohen-Macaulay.

(a) $\nu_{A}(B) \leq e$ and $\operatorname{embdim}(B) \leq e+d-1$.

(b) If $k$ is a perfect field, $A$ is a standard graded $k$-algebra, and $A \subset B$ is an extension of graded rings, then the graded $A$-module $B$ is generated in degrees at most $\max \{0, e-2\}$; in particular $\operatorname{embdeg}(B) \leq \max \{1, e-2\}$.

Proof. Let $T$ be a Noether normalization of $A$ with $\operatorname{rank}_{T} A=\operatorname{deg}(A)=e$. Being a Cohen-Macaulay ring, $B$ is also a maximal Cohen-Macaulay $T$-module. Hence the $T$-module $B$ is projective, thus free necessarily of rank $e$.

(a) The first assertion is obvious now and the second one follows because 1 is locally basic for the $T$-module $B$, forcing $B / T$ to be projective, hence free of rank $e-1$.

(b) We may assume that $k$ is an infinite field. There exists a standard graded subring $S=T[t] /(f(t))$ of $A$ with $f(t)$ a monic polynomial in $t$ of degree $e$; see for instance Proposition 5.5. Notice that $S$ is a graded free $T$-module of rank $e$ generated in degrees at most $e-1$. Consider the exact sequence of graded $T-$ modules,

$$
0 \rightarrow S \longrightarrow B \longrightarrow C \rightarrow 0
$$

Since $B$ is free, $S$ is a syzygy-module of $C$. Thus a syzygy-module of $C$ is generated in degrees at most $e-1$. But $C$ has rank zero and hence does not have a nontrivial free summand. It follows that the graded $T$-module $C$ is generated in degrees at most $e-2$. Hence as a graded $S$-module, $B$ is generated in degrees at most $\max \{0, e-2\}$, and our assertions follow.

The Cohen-Macaulay assumption in Theorem 2.1 is satisfied for $B=\bar{A}$ if $d \leq 2$ or $\operatorname{deg}(A) \leq 2$, and by a theorem of Hochster ([13, Theorem 1]), if the algebra $A$ is generated by monomials in a polynomial ring over $k$. For non-birational extensions however, it can easily fail to hold. Here is a partial explanation:

Proposition 2.2. Let $A$ be an affine normal domain over a field of characteristic zero. If $A$ is not Cohen-Macaulay, then any finite extension $B$ which is a domain is not Cohen-Macaulay either.

Proof. Let $K \subset L$ be the quotient fields of $A$ and $B$, respectively. The trace map $\operatorname{tr}_{L / K}$ induces an $A$-linear map

$$
\frac{1}{s} \operatorname{tr}_{L / K}: B \mapsto A,
$$

where $s$ is the relative degree $[L: K]$ of the field extension. It provides a splitting of $A$-modules

$$
B=A \oplus M .
$$

On the other hand, for every prime ideal $\mathfrak{p}$ of $A$ we have the standard inequality for the depth of direct summands

$$
\operatorname{depth}_{A_{\mathfrak{p}}} B_{\mathfrak{p}} \leq \operatorname{depth} A_{\mathfrak{p}},
$$

and then $B$ cannot be a Cohen-Macaulay ring.

An example of such rings is built as follows. Let $k$ be a field of characteristic zero, let $R=k[x, y, z] /(f)$, say $f=x^{3}+y^{3}+z^{3}$, and let $A$ be the Rees algebra of $(x, y, z) R$. It is easy to see that $A$ is normal, but not Cohen-Macaulay. 


\section{Dimension $3^{++}$}

The case of $\operatorname{dim} A \leq 2$ or $\operatorname{deg}(A) \leq 2$ being considered above, we focus here on conditions that are always satisfied when $\operatorname{dim} A=3$ and $\operatorname{deg}(A) \geq 3$. Because of their dependence on Jacobian ideals most of our assertions will only be valid over fields of characteristic zero.

Let $A$ be an affine algebra over a field $k$, of dimension $d$ with integral closure $B=\bar{A}$. We write $\mathfrak{c}(A)$ for the conductor $A:_{A} B$ of $A$. If $\Omega_{k}(A)$ is the module of Kähler differentials of $A$ over $k$, we denote by $J(A)$ the Jacobian ideal Fitt $_{d}\left(\Omega_{k}(A)\right)$ of $A$ over $k$. Recall that $V(J(A))=\operatorname{Sing}(A)$ in case $k$ is perfect and $A$ is reduced and equidimensional. Under the same assumptions one has $J(A) \subset \mathfrak{c}(A)$ by 20 . Theorem 2] (see also [21] or [2] 3.1]). We write $\operatorname{deg}(\cdot)$ for the multiplicity of finite graded modules over standard graded $k$-algebras.

Lemma 3.1. Let $k$ be a field of characteristic zero, let $S$ be a reduced and equidimensional standard graded $k$-algebra of dimension $d$ and multiplicity $e$ and assume that $\operatorname{embdim}(S) \leq d+1$. Let $S \subset B$ be a finite and birational extension of graded rings.

(a) The $S$-module $B / S$ satisfies

$$
\operatorname{deg}(B / S) \leq(e-1)^{2}
$$

If $e \geq 3$, then

$$
\operatorname{deg}(B / S) \leq(e-1)^{2}-1 .
$$

If $e \geq 4$ or if $B$ is $S_{2}$, but not Cohen-Macaulay, then

$$
\operatorname{deg}(B / S) \leq(e-1)^{2}-2 .
$$

(b) If $B$ is Cohen-Macaulay, then

$$
\operatorname{deg}(B / S) \leq\left(\begin{array}{l}
e \\
2
\end{array}\right)
$$

If, moreover, $k$ is algebraically closed and $S$ is a domain, then

$$
\operatorname{deg}(B / S) \leq\left(\begin{array}{c}
e-1 \\
2
\end{array}\right) .
$$

Proof. (a) We may assume that $S \neq B$. As $S$ satisfies $S_{2}$ and the extension $S \subset B$ is finite and birational, it follows that the $S$-module $B / S$ is of codimension one. Thus, since $S$ is Gorenstein,

$$
\operatorname{deg}(B / S)=\operatorname{deg}\left(\operatorname{Ext}_{S}^{1}(B / S, S)\right) .
$$

Applying $\operatorname{Hom}_{S}(\cdot, S)$ to the exact sequence

$$
0 \rightarrow S \longrightarrow B \longrightarrow B / S \rightarrow 0
$$

yields an exact sequence

$$
0 \rightarrow S / S:{ }_{S} B \longrightarrow \operatorname{Ext}_{S}^{1}(B / S, S) \longrightarrow \operatorname{Ext}_{S}^{1}(B, S) \rightarrow 0 .
$$

Since $B$ is torsionfree over the Gorenstein ring $S$, the $S$-module $\operatorname{Ext}_{S}^{1}(B, S)$ has codimension at least 2 . Thus

$$
\operatorname{deg}\left(\operatorname{Ext}_{S}^{1}(B / S, S)\right)=\operatorname{deg}\left(S / S:_{S} B\right) .
$$

On the other hand write $S=R /(f)$ with $R=k\left[x_{1}, \ldots, x_{n}\right]$ a polynomial ring and $f$ a form of degree $e$. Consider the $R$-ideal $J=\left(\frac{\partial f}{\partial x_{1}}, \ldots, \frac{\partial f}{\partial x_{n}}\right)$. One has 
$f \in J$ since char $k=0$, and therefore $J /(f)=J(S)$. In particular $J$ is an $R_{-}$ ideal of height at least 2. As $J$ is generated by forms of degree $e-1$, there exist homogeneous polynomials $g, h$ in $J$ of degree $e-1$, so that $g, h$ and $f, h$ are regular sequences. Write $K$ for the preimage of $S:_{S} B$ in $R$, which is an $R$-ideal of height 2. One has $J(S) \subset \mathfrak{c}(S)=S:_{S} \bar{S}$ by [20, Theorem 2] (see also [21] or [2, 3.1]). Hence $J(S) \subset S: S B$, which implies $J \subset K$. Consequently, the regular sequences $g, h$ and $f, h$ are contained in the height 2 ideal $K$. Therefore

$$
\begin{aligned}
\operatorname{deg}\left(S / S:_{S} B\right)=\operatorname{deg}(R / K) & =\operatorname{deg}(R /(g, h))-\operatorname{deg}\left(R /(g, h):_{R} K\right) \\
& =(e-1)^{2}-\operatorname{deg}\left(R /(g, h):_{R} K\right) .
\end{aligned}
$$

Suppose that $\operatorname{deg}\left(R /(g, h):_{R} K\right)$ is 0 or 1 , respectively. In this case the ideal $(g, h):_{R} K$ is the unit ideal or is generated by linear forms, hence the double link $(f, h):_{R} K$ contains a linear or a quadratic form, respectively. On the other hand

$$
\left((f, h):_{R} K\right) /(f)=h S:_{S}\left(S:_{S} B\right) \subset h \bar{S} .
$$

Since the nonzero homogeneous elements of $h \bar{S}$ have degrees at least $e-1$, it would follow that $e \leq 2$ or $e \leq 3$, respectively. Furthermore, if $B$ is $S_{2}$, then $h S:_{S}\left(S:_{S} B\right)=h B$, hence $B[-(e-1)] \cong\left((f, h):_{R} K\right) /(f)$. But the latter is a Cohen-Macaulay $S$-module because the $R$-ideal $(g, h):_{R} K$ is perfect. Thus $B$ would be a Cohen-Macaulay ring.

(b) We use the same notation as in the proof of Theorem 2.1(b). In particular we consider the exact sequence of graded $T$-modules,

$$
0 \rightarrow S \stackrel{\phi}{\longrightarrow} B \longrightarrow C \rightarrow 0 .
$$

Since $S$ and $B$ are graded free $T$-modules of rank $e$, it follows that $\operatorname{deg}_{T}(C)=$ $\operatorname{deg}(T /(\operatorname{det}(\phi)))$, which equals the degree of the form $\operatorname{det}(\phi)$. Hence

$$
\operatorname{deg}_{S}(B / S)=\operatorname{deg}_{T}(C)=\operatorname{deg} \operatorname{det}(\phi) .
$$

However, $S \cong \bigoplus_{i=0}^{e-1} T(-i)$ and $B \cong \bigoplus_{i=0}^{e-1} T\left(-a_{i}\right)$ with $a_{i} \geq 0$. Hence the degree of $\operatorname{det}(\phi)$ is at most

$$
1+2+\cdots+(e-1)=\left(\begin{array}{l}
e \\
2
\end{array}\right)
$$

in the first case.

If $k$ is algebraically closed and $S$ is a domain, then only one $a_{i}$ is zero so that the degree of $\operatorname{det}(\phi)$ is bounded by

$$
(1-1)+(2-1)+\cdots+(e-1-1)=\left(\begin{array}{c}
e-1 \\
2
\end{array}\right)
$$

as asserted.

Theorem 3.2. Let $k$ be a field of characteristic zero and let $A$ be a reduced and equidimensional standard graded $k$-algebra of dimension $d$ and multiplicity $e$. If $A \subset B$ is a finite and birational extension of graded rings with $\operatorname{depth}_{A} B \geq d-1$, then

$$
\nu_{A}(B) \leq(e-1)^{2}+1
$$

and

$$
\operatorname{embdim}(B) \leq(e-1)^{2}+d+1 .
$$


Proof. There exists a homogeneous subalgebra $S$ of $A$ so that $\operatorname{embdim}(S) \leq d+1$ and the extension $S \subset A$ is finite and birational; see for instance Proposition 5.5. Notice that $\operatorname{deg}(S)=\operatorname{deg}(A)=e$. The $S$-module $B / S$ is Cohen-Macaulay. Therefore $\nu_{S}(B / S) \leq \operatorname{deg}(B / S)$ and the assertions follow from Lemma 3.1(a).

Remark 3.3. Theorem 2.1(a) and Lemma 3.1 show that the estimates of Theorem 3.2 can be sharpened under suitable additional assumptions. Indeed, if $e \geq 3$, then

$$
\nu_{A}(B) \leq(e-1)^{2} \text { and } \operatorname{embdim}(B) \leq(e-1)^{2}+d .
$$

If $e \geq 4$ or if $B$ satisfies $S_{2}$ and $e \geq 3$, then

$$
\nu_{A}(B) \leq(e-1)^{2}-1 \text { and } \operatorname{embdim}(B) \leq(e-1)^{2}+d-1 .
$$

Corollary 3.4. Let $k$ be a field of characteristic zero and let $A$ be a reduced and equidimensional standard graded $k$-algebra of dimension 3 and multiplicity $e \geq 3$. The integral closure $B=\bar{A}$ satisfies

$$
\operatorname{embdim}(B) \leq(e-1)^{2}+2
$$

Remark 3.5. If in the setting of Theorem 3.2 and its proof, $T$ is a homogeneous Noether normalization of $A$, then $\nu_{T}(B) \leq e(e-1)^{2}+e$ as can be seen by applying the theorem with $A=S$. This bound is not strictly module theoretic, the algebra structure of $B$ really matters. The assertion fails if $B$ is merely a finite $T$-module, even a graded reflexive $T$-module, with $\operatorname{depth}_{T} B \geq d-1$. For example, let $T$ be a polynomial ring in $d$ variables over an infinite field and $I$ a homogeneous perfect $T$ ideal of height 2 that is generically a complete intersection, but has a large number of generators (such ideals exist whenever $d \geq 3$ ). There is an exact sequence of the form

$$
0 \rightarrow T(-a) \longrightarrow E \longrightarrow I \rightarrow 0,
$$

with $E$ a graded reflexive $T$-module. Now indeed $\operatorname{depth} E=d-1$ and $\operatorname{deg}(E)=2$, whereas $\nu_{T}(E) \gg 0$.

\section{Non-HomogeneOUS ALgebras}

We now formulate a version of the estimates of the previous section valid for general affine algebras. We will deal with the non-homogeneous version of Lemma 3.1 and Theorem 3.2. Here we write $\operatorname{deg}(\cdot)$ for the multiplicity of finite modules over Noetherian local rings.

Proposition 4.1. Let $R=k\left[x_{1}, \ldots, x_{d+1}\right]$ be a polynomial ring over a perfect field $k$, let $f, g$ be an $R$-regular sequence, and let $\mathfrak{p}$ be a prime ideal of $R$.

(a) If the degrees of $f$ and $g$ in the variables $x_{1}, \ldots, x_{d+1}$ are $m$ and $n$, then

$$
\operatorname{deg}\left((R /(f, g))_{\mathfrak{p}}\right) \leq m n .
$$

(b) If the degrees of $f$ and $g$ in the variables $x_{1}, \ldots, x_{d}$ are $m^{\prime}$ and $n^{\prime}$, the degrees of $f$ and $g$ in $x_{d+1}$ are $m^{\prime \prime}$ and $n^{\prime \prime}$, and $g$ is monic in $x_{d+1}$, then

$$
\operatorname{deg}\left((R /(f, g))_{\mathfrak{p}}\right) \leq\left(m^{\prime} n^{\prime \prime}+m^{\prime \prime} n^{\prime}\right) n^{\prime \prime} .
$$

Proof. We may assume that $\mathfrak{p}$ is a maximal ideal containing $f, g$, and after passing to the algebraic closure of $k$ we may suppose that $\mathfrak{p}=\left(x_{1}, \ldots, x_{d+1}\right)$. 
To prove (a) let $z$ be a new variable, $\widetilde{R}=R[z], \widetilde{\mathfrak{p}}=\mathfrak{p} \widetilde{R}$, and $\widetilde{f}, \widetilde{g}$ the homogenizations of $f$ and $g$ with respect to $z$. Notice that $\widetilde{f}, \widetilde{g}$ are homogeneous of degrees $m, n$ and form a regular sequence in $\widetilde{R}$. Thus $\operatorname{deg}(\widetilde{R} /(\widetilde{f}, \widetilde{g}))=m n$. On the other hand, $\widetilde{\mathfrak{p}}$ is contained in the homogeneous maximal ideal of $\widetilde{R}$ and $(\widetilde{R} /(\widetilde{f}, \widetilde{g}))_{\tilde{\mathfrak{p}}} \cong(\widetilde{R} /(\widetilde{f}, \widetilde{g}))_{(\widetilde{\mathfrak{p}})}(z)$. Since $(\widetilde{R} /(\widetilde{f}, \widetilde{g}))_{(\widetilde{\mathfrak{p}})} \cong(R /(f, g))_{\mathfrak{p}}$, we conclude that $\operatorname{deg}\left((R /(f, g))_{\mathfrak{p}}\right) \leq m n$.

To prove (b) write $T=k\left[x_{1}, \ldots, x_{d}\right], \mathfrak{q}=\mathfrak{p} \cap T$, and let $h$ be the resultant of the polynomials $f, g$ with respect to the variable $x_{d+1}$. Now $h \neq 0$ since $f$ and $g$ form a regular sequence, $h \in(f, g) \cap T$, and $h$ has degree at most $m^{\prime} n^{\prime \prime}+m^{\prime \prime} n^{\prime}$ in the variables $x_{1}, \ldots, x_{d}$. Thus $\operatorname{deg}\left((T /(h))_{\mathfrak{q}}\right) \leq m^{\prime} n^{\prime \prime}+m^{\prime \prime} n^{\prime}$. Furthermore, $(R /(f, g))_{\mathfrak{q}}$ is a module over $(T /(h))_{\mathfrak{q}}$ whose dimension is maximal and whose number of generators is at most $n^{\prime \prime}$. Thus $\operatorname{deg}\left((R /(f, g))_{\mathfrak{q}}\right) \leq\left(m^{\prime} n^{\prime \prime}+m^{\prime \prime} n^{\prime}\right) n^{\prime \prime}$. Finally, the multiplicity of the ring $(R /(f, g))_{\mathfrak{p}}$ cannot exceed that of the module $(R /(f, g))_{\mathfrak{q}}$.

Lemma 4.2. Let $k$ be a field of characteristic zero, let $R=k\left[x_{1}, \ldots, x_{d+1}\right]$ be a polynomial ring and $f \neq 0$ a squarefree polynomial, and write $S=R /(f)$. Let $S \subset B$ be a finite and birational extension of rings and let $\mathfrak{p}$ be a prime ideal of $R$.

(a) If the degree of $f$ in the variables $x_{1}, \ldots, x_{d+1}$ is $e$, then

$$
\operatorname{deg}(B / S)_{\mathfrak{p}} \leq e(e-1) .
$$

(b) If the degree of $f$ in the variables $x_{1}, \ldots, x_{d}$ is $e^{\prime}$ and $f$ is monic in $x_{d+1}$ of degree $e^{\prime \prime}$, then

$$
\operatorname{deg}(B / S)_{\mathfrak{p}} \leq e^{\prime}\left(e^{\prime \prime}-1\right)\left(2 e^{\prime \prime}-1\right)
$$

Proof. In part (a) we may assume $e \geq 1$ and apply a linear change of variables to suppose that $f$ is monic in $x_{d+1}$. Thus in either case, the homomorphism $k\left[x_{1}, \ldots, x_{d}\right] \rightarrow S$ is finite and generically smooth. Therefore $f$ and its derivative $f^{\prime}=\frac{\partial f}{\partial x_{d+1}}$ generate the unit ideal or form a regular sequence in $R$. Applying Proposition 4.1(a) we conclude that $\operatorname{deg}\left(\left(R /\left(f, f^{\prime}\right)\right)_{\mathfrak{p}}\right) \leq e(e-1)$ in part (a), and from Proposition 4.1 (b) we obtain

$$
\operatorname{deg}\left(\left(R /\left(f, f^{\prime}\right)\right)_{\mathfrak{p}}\right) \leq\left(e^{\prime}\left(e^{\prime \prime}-1\right)+e^{\prime \prime} e^{\prime}\right)\left(e^{\prime \prime}-1\right)=e^{\prime}\left(e^{\prime \prime}-1\right)\left(2 e^{\prime \prime}-1\right)
$$

in the setting of $(b)$.

Now one proceeds as in the proof of Lemma 3.1(a).

Theorem 4.3. Let $k$ be a field of characteristic zero and let $A$ be a reduced and equidimensional affine $k$-algebra of dimension $d$. Let $S$ be a $k$-subalgebra of $A$ with $\operatorname{embdim}(S) \leq d+1$ so that the extension $S \subset A$ is finite and birational, and write $S=k\left[x_{1}, \ldots, x_{d+1}\right] /(f)$. Further let $A \subset B$ be a finite and birational extension of rings with $S \neq B$ satisfying $\operatorname{depth}_{A_{\mathfrak{p}}} B_{\mathfrak{p}} \geq d-1$ for every maximal ideal $\mathfrak{p}$ of $A$.

(a) If the degree of $f$ in the variables $x_{1}, \ldots, x_{d+1}$ is e, then

$$
\nu_{A}(B) \leq e(e-1)+d-1
$$

and

$$
\operatorname{embdim}(B) \leq e(e-1)+2 d-1 .
$$

(b) If the degree of $f$ in the variables $x_{1}, \ldots, x_{d}$ is $e^{\prime}$ and $f$ is monic in $x_{d+1}$ of degree $e^{\prime \prime}$, then

$$
\operatorname{embdim}(B) \leq e^{\prime}\left(e^{\prime \prime}-1\right)\left(2 e^{\prime \prime}-1\right)+2 d-1 .
$$


Proof. In either case we may assume that $f$ is monic in $x_{d+1}$. Using Lemma 4.2 one proceeds as in the proof of Theorem 3.2 to obtain estimates for the local numbers of generators $\nu_{S_{\mathfrak{p}}}\left((B / S)_{\mathfrak{p}}\right)$ with $\mathfrak{p}$ any prime ideal of $S$. Also notice that locally in codimension one in $T=k\left[x_{1}, \ldots, x_{d}\right]$, the $T$-module $B / T$ is free of rank at most $e-1$ for (a) and of rank $e^{\prime \prime}-1$ for (b). Now the appropriate global bounds for $\nu_{S}(B / S)$ follow from Forster's theorem ([10] Satz 1]).

Remark 4.4. Let $A$ be an affine $k$-algebra and write $A=R / I$ with $R$ a polynomial ring over $k$. For $z$ a new variable let $\widetilde{I}$ denote the homogenization of $I$ in $\widetilde{R}=R[z]$ and set $\widetilde{A}=\widetilde{R} / \widetilde{I}$. The integer $e$ occurring in the estimate of Theorem 4.3(a) satisfies the inequality $e \geq \operatorname{deg}(A)$ and, for a suitable choice of $S$, the equality $e=\operatorname{deg}(\widetilde{A})$. Unlike Theorems 2.1(a) and 3.2, the assertion of the present theorem is no longer true with $\operatorname{deg}(A)$ in place of $e$, even if $A$ is a positively graded $k^{-}$ algebra. In fact it is impossible to estimate the embedding dimension of $B$ only in terms of the dimension and the degree of $A$, unless $A$ is standard graded or $B$ is Cohen-Macaulay.

For example let $T$ be a polynomial ring in $d \geq 2$ variables over a field $k$, let $u \in T$ be a quadratic form which is not a square (which exists), and let $I \neq 0$ be a homogeneous $T$-ideal of projective dimension at most 1 . Write $t$ for the image of the indeterminate $z$ in the ring $C=T[z] /\left(z^{2}-u\right)$, and set $B=T \oplus I t \subset C$. Now $C$ is a standard graded domain over $k$ and $B$ is a positively graded $k$-subalgebra of $C$ (with integral closure $C$ if char $k \neq 2$ and $u$ is squarefree). The assumptions of Theorem 4.3(a) are satisfied for $A=B$ (if $\operatorname{char} k=0$ ). Notice that $\operatorname{deg}(A)=2$, whereas $\operatorname{embdim}(B)=n+d$ with $n=\nu(I)$, which can be arbitrarily large. We compare this to the prediction of Theorem 4.3, if $I$ has height 2 and a linear presentation matrix (and $\operatorname{char} k=0$ ). In this case one can choose $e=e^{\prime}=2 n$ and $e^{\prime \prime}=2$. Therefore parts (a) and (b) of the theorem give the estimates embdim $(B) \leq 2 n(2 n-1)+2 d-1$ and $\operatorname{embdim}(B) \leq 6 n+2 d-1$, respectively. Also note the significance of the $S_{2}$ condition of Serre for $B$ : It forces $I$ to be a divisorial ideal and hence implies $\operatorname{embdim}(B)=d+1$, contrasting the example of Remark 3.5.

\section{Serre CONDition $R_{\ell}(\ell \geq 1)$}

Let $A$ be a reduced and equidimensional affine algebra over a perfect field $k$, let $J=J(A)$ be its Jacobian ideal and $B=\bar{A}$ its integral closure. In this section we consider the case where the singular locus of $A$ is suitably small.

Suppose that height $J \geq 2$, a condition equivalent to $A_{\mathfrak{p}}=B_{\mathfrak{p}}$ for every prime ideal $\mathfrak{p}$ of $A$ with $\operatorname{dim} A_{\mathfrak{p}}=1$. This means that $B$ is the $S_{2}$-ification of $A$, and one may describe $B$ as the outcome of a single colon operation in the total ring of quotients of $A$ (see [27, Theorem 6.4.5] for a discussion):

Theorem 5.1. Let $k$ be a perfect field and let $A$ be a reduced and equidimensional affine $k$-algebra with $J=J(A)$. If $A$ satisfies the condition $R_{1}$, its integral closure $B=\bar{A}$ is given by

$$
B=A: J
$$

The issue is to estimate how many generators this process requires. One of our results, for $d=4$, will do precisely this. For higher dimensions we shall require a condition of the order $R_{d-3}$. Before we can get to this we need to examine various "approximations" of $A$ by some more tractable subrings much in the manner that 
the hypersurface subring $S$ was used in Section 3. They will be aimed at converting information about the degrees of the generators of a "thick" portion of the Jacobian ideal $J$ in terms of the multiplicity of $A$. If $(S, \mathfrak{n})$ is a Noetherian local ring, we write $\operatorname{embdim}(S)=\nu_{S}(\mathfrak{n})$ and $\operatorname{ecodim}(S)=\operatorname{embdim}(S)-\operatorname{dim} S$.

Proposition 5.2. Let $k$ be an infinite perfect field, let $A=k\left[y_{1}, \ldots, y_{n}\right]$ be an affine $k$-algebra of dimension $d$, let $\mathfrak{p}$ be a prime ideal of $A$, and let $g \geq 1$ be an integer with $d+g \geq \operatorname{embdim}\left(A_{\mathfrak{p}}\right)+\operatorname{dim} A / \mathfrak{p}$. Let $x_{1}, \ldots, x_{d+g}$ be general $k$-linear combinations of $y_{1}, \ldots, y_{n}$ and write $S=k\left[x_{1}, \ldots, x_{d+g}\right]$. Then the extension $S \subset A$ is finite and

$$
S_{\mathfrak{p} \cap S}=A_{\mathfrak{p} \cap S}=A_{\mathfrak{p}}
$$

If $A$ is reduced and equidimensional, then the extension $S \subset A$ is also birational.

Proof. The assertion about finiteness is obvious. The claim about birationality follows from the above equalities applied to the minimal primes of $A$.

Set $\mathfrak{q}=\mathfrak{p} \cap S$. To show the equalities $S_{\mathfrak{q}}=A_{\mathfrak{q}}=A_{\mathfrak{p}}$, write $k(\mathfrak{p})$ for the residue field of $\mathfrak{p}$ and consider the exact sequence

$$
0 \rightarrow \mathbb{D} \longrightarrow R=k(\mathfrak{p}) \otimes_{k} A \longrightarrow k(\mathfrak{p}) \rightarrow 0
$$

induced by the multiplication map $A \otimes_{k} A \rightarrow A$. Notice that

$$
\mathbb{D}=\sum_{i=1}^{n} R\left(\overline{y_{i}} \otimes 1-1 \otimes y_{i}\right)
$$

is a maximal ideal of $R$. Since

$$
\nu\left(\mathbb{D}_{\mathbb{D}}\right) \leq \nu\left(\Omega_{k}\left(A_{\mathfrak{p}}\right)\right) \leq \operatorname{embdim}\left(A_{\mathfrak{p}}\right)+\operatorname{dim} A / \mathfrak{p} \leq d+g,
$$

one has $\mathbb{D}_{\mathbb{D}}=\sum_{i=1}^{d+g} R_{\mathbb{D}}\left(\overline{x_{i}} \otimes 1-1 \otimes x_{i}\right)$. On the other hand, since $\operatorname{dim} R=$ $d<d+g$, it follows that $\mathbb{D}=\sqrt{\sum_{i=1}^{d+g} R\left(\overline{x_{i}} \otimes 1-1 \otimes x_{i}\right)}$; to see this recall that every ideal in a $d$-dimensional ring containing an infinite field $k$ is generated up to radical by $d+1$ general $k$-linear combinations of its generators. It follows that $\mathbb{D}=\sum_{i=1}^{d+g} R\left(\overline{x_{i}} \otimes 1-1 \otimes x_{i}\right)$. Thus by (1),$k(\mathfrak{p}) \otimes_{S} A \cong k(\mathfrak{p})$. Comparing numbers of generators over the ring $S_{\mathfrak{q}}$ we conclude that $\nu_{S_{\mathfrak{q}}}\left(A_{\mathfrak{q}}\right)=1$, hence $S_{\mathfrak{q}}=A_{\mathfrak{q}}$. In particular $A_{\mathfrak{q}}$ is local and we also obtain $A_{\mathfrak{q}}=A_{\mathfrak{p}}$.

Corollary 5.3. Let $k$ be an infinite perfect field and let $A$ be a reduced and equidimensional standard graded $k$-algebra of dimension $d$. Write $\mathcal{A}$ for the set of all homogeneous subalgebras $S=k\left[x_{1}, \ldots, x_{d+1}\right]$ generated by linear forms of $A$ so that the extension $S \subset A$ is finite and birational, and set $\mathcal{J}=\sum_{S \in \mathcal{A}} A \cdot J(S)$. Then $\sqrt{J(A)}=\sqrt{\mathcal{J}}$.

Proof. First notice that every $S \in \mathcal{A}$ is again reduced and equidimensional. Let $\mathfrak{p}$ be a fixed prime ideal of $A$. Applying Proposition 5.2 with $g=1$ we see that $A_{\mathfrak{p}}$ is regular if and only if $S_{\mathfrak{p} \cap S}$ is regular for some $S \in \mathcal{A}$. Equivalently, $J(A) \subset \mathfrak{p}$ if and only if $J(S) \subset \mathfrak{p} \cap S$ for every $S \in \mathcal{A}$, if and only if $A \cdot J(S) \subset \mathfrak{p}$ for every $S \in \mathcal{A}$.

Corollary 5.4. Let $k$ be an infinite perfect field and let $A$ be a reduced and equidimensional standard graded $k$-algebra with $e=\operatorname{deg}(A)$ and $c=\operatorname{codim}(\operatorname{Sing}(A))$. Then the conductor of $A$ contains an ideal of height $c$ generated by forms of degree $e-1$. 
Proof. We claim that the ideal $\mathcal{J}$ of Corollary 5.3 has the desired properties. First, by the corollary, height $\mathcal{J}=c$. Now let $S \in \mathcal{A}$. Since $S=k\left[x_{1}, \ldots, x_{d+1}\right]$ is reduced and equidimensional of dimension $d$, we can write $S=k\left[X_{1}, \ldots, X_{d+1}\right] /(f)$ where $f$ is a form. As the homogeneous extension $S \subset A$ is finite and birational, one has $\operatorname{deg}(S)=\operatorname{deg}(A)$, thus $\operatorname{deg} f=e$. Therefore $J(S)$ is generated by forms of degree $e-1$ and hence $\mathcal{J}$ has the same property. Finally by [20, Theorem 2], $J(S)$ is contained in the conductor $\mathfrak{c}(S)$ of $S$. But $\mathfrak{c}(S) \subset \mathfrak{c}(A)$ since the extension $S \subset A$ is finite and birational. Thus indeed $\mathcal{J} \subset \mathfrak{c}(A)$.

The proof of the main theorem in this section requires another preparatory result akin to Proposition 5.2, a generic projection lemma that is known in a geometric context, at least for algebras with small singular locus:

Proposition 5.5. Let $k$ be an infinite perfect field, let $A=k\left[y_{1}, \ldots, y_{n}\right]$ be an equidimensional $k$-algebra of dimension $d$, and let $s \geq 0$ be an integer. Let $x_{1}, \ldots$, $x_{d+s}$ be general $k$-linear combinations of $y_{1}, \ldots, y_{n}$ and write $S=k\left[x_{1}, \ldots, x_{d+s}\right] \subset$ $A$. If $\operatorname{ecodim}\left(A_{\mathfrak{p}}\right) \leq \operatorname{dim} A_{\mathfrak{p}}$ for every prime $\mathfrak{p}$ of $A$ with $\operatorname{dim} A_{\mathfrak{p}} \leq s-1$, then $S_{\mathfrak{q}}=A_{\mathfrak{q}}$ for every prime $\mathfrak{q}$ of $S$ with $\operatorname{dim} S_{\mathfrak{q}} \leq s-1$.

Proof. Consider the exact sequence

$$
0 \rightarrow \mathbb{D} \longrightarrow R=A \otimes_{k} A \stackrel{\text { mult }}{\longrightarrow} A \rightarrow 0,
$$

where $\mathbb{D}=\sum_{i=1}^{n} R\left(y_{i} \otimes 1-1 \otimes y_{i}\right)$. By our assumption and [23, 2.2(b)], $\nu\left(\mathbb{D}_{Q}\right) \leq$ $\operatorname{dim} R_{Q}$ for every $Q \in V(\mathbb{D})$ with $\operatorname{dim} R_{Q} \leq d+s-1$, i.e., $\mathbb{D}$ satisfies the condition $G_{d+s}$ from [1. p. 312]. But then according to [1, the proof of 2.3],

$$
\text { height }\left(\sum_{i=1}^{d+s} R\left(x_{i} \otimes 1-1 \otimes x_{i}\right)\right): \mathbb{D} \geq d+s \text {. }
$$

Now let $\mathfrak{q}$ be a prime of $S$ with $\operatorname{dim} S_{\mathfrak{q}} \leq s-1$. As $\operatorname{dim} A_{\mathfrak{q}} \otimes_{k} A_{\mathfrak{q}} \leq \operatorname{dim} A_{\mathfrak{q}}+$ $\operatorname{dim} A \leq d+s-1$, (3) implies that

$$
\left(A_{\mathfrak{q}} \otimes_{k} A_{\mathfrak{q}}\right) \mathbb{D}=\sum_{i=1}^{d+s}\left(A_{\mathfrak{q}} \otimes_{k} A_{\mathfrak{q}}\right)\left(x_{i} \otimes 1-1 \otimes x_{i}\right) .
$$

Thus $A_{\mathfrak{q}} \otimes_{S_{\mathfrak{q}}} A_{\mathfrak{q}} \cong A_{\mathfrak{q}}$ by (2). Since $A_{\mathfrak{q}}$ is a finite $S_{\mathfrak{q}}$-module, comparing numbers of generators then yields $\nu_{S_{\mathfrak{q}}}\left(A_{\mathfrak{q}}\right)=1$, hence $S_{\mathfrak{q}}=A_{\mathfrak{q}}$.

Theorem 5.6. Let $k$ be a field of characteristic zero and let $A$ be a reduced and equidimensional standard graded $k$-algebra of dimension $d \geq 3$ and multiplicity e. Let $A \subset B$ be a finite and birational extension of graded rings and write $t=$ $\min \left\{d-2, \operatorname{depth}_{A} B\right\}$. If $A$ satisfies $R_{d-t-1}$, then

$$
\nu_{A}(B) \leq 1 / 2(e(e-1))^{2^{d-t-1}}-1 / 2(e(e-1))^{2^{d-t-2}}+1
$$

and

$$
\operatorname{embdim}(B) \leq 1 / 2(e(e-1))^{2^{d-t-1}}-1 / 2(e(e-1))^{2^{d-t-2}}+2 d-t .
$$

Proof. By Proposition 5.5 we may replace $A$ by a suitable $k$-subalgebra to assume that $\operatorname{embdim}(A) \leq 2 d-t$. Thus it suffices to show that

$$
\nu_{A}(B / A) \leq 1 / 2(e(e-1))^{2^{d-t-1}}-1 / 2(e(e-1))^{2^{d-t-2}} .
$$

Let $\mathbf{x}=x_{1}, \ldots, x_{t-1}$ be a sequence of general linear forms in $A$. Notice that $\mathbf{x}$ forms a regular sequence on $B$. Write $B^{\prime}=B /(\mathbf{x}) B$ and $A^{\prime}$ for the image of $A$ in $B^{\prime}$. 
Since $\operatorname{dim}_{A} B / A \leq t$, one has $\operatorname{dim}_{A^{\prime}} B^{\prime} / A^{\prime}=\operatorname{dim}_{A} B^{\prime} / A^{\prime} \leq 1$. Furthermore by 9 . 4.7], $B^{\prime}$ satisfies $R_{d-t-1}$, and is reduced and equidimensional of dimension $d-t+1 \geq$ 3 . Thus the standard graded $k$-algebra $A^{\prime}$ has the same properties. Moreover, $\operatorname{deg}\left(A^{\prime}\right)=\operatorname{deg}_{A^{\prime}}\left(B^{\prime}\right)=\operatorname{deg}_{A}(B)=\operatorname{deg}(A)=e$. By the graded Nakayama lemma, as $B^{\prime} / A^{\prime} \subset \overline{A^{\prime}} / A^{\prime}$, it suffices to show that for every $A^{\prime}$-submodule $C$ of $\overline{A^{\prime}} / A^{\prime}$,

$$
\nu_{A^{\prime}}(C) \leq 1 / 2\left(\operatorname{deg}\left(A^{\prime}\right)\left(\operatorname{deg}\left(A^{\prime}\right)-1\right)\right)^{2^{\operatorname{dim} A^{\prime}-2}}-1 / 2\left(\operatorname{deg}\left(A^{\prime}\right)\left(\operatorname{deg}\left(A^{\prime}\right)-1\right)\right)^{2^{\operatorname{dim} A^{\prime}-3}} .
$$

Changing notation we prove the following: If $A$ is a reduced and equidimensional standard graded $k$-algebra of dimension $d \geq 3$ and multiplicity $e$ satisfying $R_{d-2}$, then for every $A$-submodule $C$ of $\bar{A} / A$,

$$
\nu_{A}(C) \leq 1 / 2(e(e-1))^{2^{d-2}}-1 / 2(e(e-1))^{2^{d-3}} .
$$

Now write $B=\bar{A}$ for the integral closure and $\mathfrak{c}=\mathfrak{c}(A)$ for the conductor of $A$. By Corollary 5.4 $\mathfrak{c}$ contains forms of degree $e-1$ that generate an ideal $\mathcal{J}$ of height at least $d-1$. Let $x$ be a general $k$-linear combination of these forms. Write $B^{\prime}=B / x B$ and let $A^{\prime}$ be the image of $A$ in $B^{\prime}$. As height $\mathcal{J} \geq d-1$, it follows that $B^{\prime}$ satisfies $R_{d-3}([9,4.7])$, and $B^{\prime}$ is reduced and equidimensional of dimension $d-1 \geq 2$. Since $\operatorname{dim}_{A^{\prime}} B^{\prime} / A^{\prime} \leq 1, A^{\prime}$ then has the same properties. Notice that $\operatorname{deg}\left(A^{\prime}\right)=e(e-1)$ because $x$ is a $B$-regular form of degree $e-1$. On the other hand, as $x \in \mathfrak{c}=\operatorname{ann}_{A}(B / A)$, we have $B / A=B^{\prime} / A^{\prime}$ and therefore $C \subset B / A=B^{\prime} / A^{\prime} \subset \overline{A^{\prime}} / A^{\prime}$.

We are now ready to prove (41) by induction on $d \geq 3$. We may assume $C \neq 0$. If $d=3$, let $x_{1}, x_{2}, x_{3}$ be general linear forms in $A$, and write $S=k\left[x_{1}, x_{2}, x_{3}\right]$ and $S^{\prime}$ for the image of $S$ in $A^{\prime}$. Now $S^{\prime} \subset A^{\prime} \subset \overline{A^{\prime}}$ are finite and birational extensions of two-dimensional rings, embdim $\left(S^{\prime}\right) \leq 3=\operatorname{dim} S^{\prime}+1, \operatorname{deg}\left(S^{\prime}\right)=\operatorname{deg}\left(A^{\prime}\right)$, and $S^{\prime}$ and $\overline{A^{\prime}}$ are Cohen-Macaulay. Thus the $S^{\prime}$-module $\overline{A^{\prime}} / S^{\prime}$ is Cohen-Macaulay of dimension 1 and has multiplicity at most $\left(\underset{2}{\operatorname{deg}\left(A^{\prime}\right)}\right)$ by Lemma 3.1 (b). Let $D \subset \overline{A^{\prime}} / S^{\prime}$ be the preimage of $C \subset \overline{A^{\prime}} / A^{\prime}$ under the natural projection from $\overline{A^{\prime}} / S^{\prime}$ to $\overline{A^{\prime}} / A^{\prime}$. As an $S^{\prime}$-submodule of $\overline{A^{\prime}} / S^{\prime}, D$ is also Cohen-Macaulay of dimension 1. It follows that $\nu_{S^{\prime}}(D) \leq \operatorname{deg}(D) \leq \operatorname{deg}\left(\overline{A^{\prime}} / S^{\prime}\right)$. Therefore

$$
\begin{aligned}
\nu_{A}(C) & \leq \nu_{S^{\prime}}(C) \leq \nu_{S^{\prime}}(D) \leq \operatorname{deg}\left(\overline{A^{\prime}} / S^{\prime}\right) \\
& \leq\left(\begin{array}{c}
\operatorname{deg}\left(A^{\prime}\right) \\
2
\end{array}\right)=1 / 2(e(e-1))^{2}-1 / 2 e(e-1),
\end{aligned}
$$

establishing (4) for $d=3$.

If $d \geq 4$, then by the induction hypothesis,

$$
\begin{aligned}
\nu_{A}(C) & =\nu_{A^{\prime}}(C) \\
& \leq 1 / 2\left(\operatorname{deg}\left(A^{\prime}\right)\left(\operatorname{deg}\left(A^{\prime}\right)-1\right)\right)^{2^{\operatorname{dim} A^{\prime}-2}}-1 / 2\left(\operatorname{deg}\left(A^{\prime}\right)\left(\operatorname{deg}\left(A^{\prime}\right)-1\right)\right)^{2^{\operatorname{dim} A^{\prime}-3}} \\
& =1 / 2(e(e-1)(e(e-1)-1))^{2^{d-3}}-1 / 2(e(e-1)(e(e-1)-1))^{2^{d-4}} \\
& \leq 1 / 2(e(e-1))^{2^{d-2}}-1 / 2(e(e-1))^{2^{d-3}} .
\end{aligned}
$$

To see the last inequality set $a=e(e-1)$ and $\alpha=2^{d-4}$, and notice that $(a(a-1))^{2 \alpha}-(a(a-1))^{\alpha} \leq a^{4 \alpha}-a^{2 \alpha}$.

Corollary 5.7. Let $k$ be a field of characteristic zero and let $A$ be a reduced and equidimensional standard graded $k$-algebra of dimension $d \geq 4$ and multiplicity $e$. 
If $A$ satisfies $R_{d-3}$, then for $B=\bar{A}$,

$$
\operatorname{embdim}(B) \leq 1 / 2(e(e-1))^{2^{d-3}}-1 / 2(e(e-1))^{2^{d-4}}+2 d-2 .
$$

In particular if $d=4$ and $A$ satisfies $R_{1}$, then

$$
\operatorname{embdim}(B) \leq 1 / 2 e^{4}-e^{3}+1 / 2 e+6 .
$$

\section{BoundS ON DEGREES}

In this section we give degree bounds for the generators of the integral closure of standard graded integral domains over a field of characteristic zero. Besides being interesting in their own right, such bounds also lead to estimates of the embedding dimension, due to the following observation:

Proposition 6.1. Let $k$ be an infinite perfect field and let $A$ be a reduced and equidimensional standard graded $k$-algebra of dimension $d \geq 1$ and multiplicity $e$. Let $A \subset B$ be a finite and birational extension of graded rings.

(a) If $\operatorname{embdim}(A) \leq d+1$ and the graded $A$-module $B$ is generated in degrees at most $s$, then

$$
\nu_{A}(B) \leq\left(\begin{array}{c}
s+e+d-1 \\
d
\end{array}\right)-\left(\begin{array}{c}
s+d-1 \\
d
\end{array}\right) .
$$

(b) If $\operatorname{embdeg}(B)=s$, then

$$
\operatorname{embdim}(B) \leq\left(\begin{array}{c}
s+e+d-1 \\
d
\end{array}\right)-\left(\begin{array}{c}
s+d-1 \\
d
\end{array}\right)+d .
$$

Proof. Replacing $A$ by any $S \in \mathcal{A}$ as in Corollary [5.3 we may assume that $\operatorname{embdim}(A) \leq d+1$ in part (b) as well. Now $A=k\left[x_{1}, \ldots, x_{d+1}\right] /(f)$ with $f$ a homogeneous polynomial of degree $e$. Let $D \subset B$ be the $A$-submodule generated by all forms in $B$ of degrees $\leq s$. By Corollary [5.4 (or [20, Theorem 2]), $A: B$ contains a homogeneous element $x$ of degree $e-1$ that is regular on $A$, hence on $B$. Thus $I=x D \cong D[-(e-1)]$ is a homogeneous $A$-ideal generated by forms of degrees $\leq s+e-1$. Let $y$ be a linear form that is regular on $A$. Multiplying the elements of a homogeneous minimal generating set of $I$ by suitable powers of $y$ one sees that

$$
\nu_{A}(I) \leq \operatorname{dim}_{k} A_{s+e-1}=\left(\begin{array}{c}
s+e+d-1 \\
d
\end{array}\right)-\left(\begin{array}{c}
s+d-1 \\
d
\end{array}\right) .
$$

Now part (a) follows since in this case $B=D \cong I[e-1]$ as $A$-modules. As to (b) notice that the $A$-module $D \cong I[e-1]$ is minimally generated by a set of the form $\{1\} \cup W$, where $W$ has $\nu_{A}(I)-1$ elements. Since $W$ generates the $A$-algebra $B$, the assertion follows in this case as well.

If $A$ is a standard graded algebra over a field we write $\operatorname{reg}(A)$ for the CastelnuovoMumford regularity and ecodim $(A)=\operatorname{embdim}(A)-\operatorname{dim} A$ for the embedding codimension of $A$. Some of our bounds have a conjectural basis, as they depend on the validity of the conjecture from [8]:

Conjecture 6.2 (Eisenbud-Goto). Let $k$ be an algebraically closed field and $A$ a standard graded domain over $k$. Then the Castelnuovo-Mumford regularity of $A$ is bounded by

$$
\operatorname{reg}(A) \leq \operatorname{deg}(A)-\operatorname{ecodim}(A)
$$


The bound of (6.2) has been established in low dimensions $(\leq 7)$, often with additional restrictions.

Theorem 6.3. Let $k$ be an algebraically closed field of characteristic zero and let $A$ be a reduced and equidimensional standard graded $k$-algebra of dimension $d$ and multiplicity $e \geq 3$. Assume that $A$ satisfies $R_{2}$. If (6.2) holds in dimension $d-1$ over the field $k$, then for $B=\bar{A}$, the graded $A$-module $B$ is generated in degrees at most $e(e-2)-1$; in particular

$$
\begin{aligned}
\operatorname{embdeg}(B) & \leq e(e-2)-1 \\
\operatorname{embdim}(B) & \leq\left(\begin{array}{c}
e(e-1)+d-2 \\
d
\end{array}\right)-\left(\begin{array}{c}
e(e-2)+d-2 \\
d
\end{array}\right)+d .
\end{aligned}
$$

Proof. We first prove the assertion about the generator degrees. We may assume that $A$ is not Cohen-Macaulay since otherwise $B=A$. Thus $\operatorname{ecodim}(A) \geq 2$. We may also suppose that $A$ is a domain, as it suffices to prove our claim modulo every minimal prime of $B=\bar{A}$. By Corollary 5.4 the conductor $\mathfrak{c}(A)=A: B$ contains an ideal $\mathcal{J}$ of height $\geq 3$ generated by homogeneous elements of degree $e-1$. Let $x$ be a general form of degree $e-1$ in $\mathcal{J}$. Since height $\mathcal{J} \geq 3$ and $B$ satisfies $S_{2}$ and has characteristic zero, 9, 4.10] shows that $B^{\prime}=B / x B$ is a domain. Notice that $x B \subset$ $A$, hence $A^{\prime}=A / x B$ is a subring of $B^{\prime}$. Thus $A^{\prime}$ is a domain of dimension $d-1$. The finite extension $A^{\prime} \subset B^{\prime}$ is birational since $\operatorname{dim}_{A^{\prime}}\left(B^{\prime} / A^{\prime}\right)=\operatorname{dim}_{A}(B / A) \leq d-3$. Therefore $\operatorname{deg}\left(A^{\prime}\right)=e(e-1)$. As $e-1 \geq 2$ and $B$ is concentrated in nonnegative degrees, it follows that $\operatorname{ecodim}\left(A^{\prime}\right)=\operatorname{ecodim}(A)+1$. However, $\operatorname{ecodim}(A) \geq 2$ by the above. According to our assumption, (6.2) holds for the standard graded domain $A^{\prime}$ of dimension $d-1$. Therefore $\operatorname{reg}\left(A^{\prime}\right) \leq e(e-1)-3$.

Let $R$ be a polynomial ring over $k$ mapping onto $A$ and $\mathfrak{P}$ a prime ideal of $R$ with $A^{\prime} \cong R / \mathfrak{P}$. The homogeneous $R$-ideal $\mathfrak{P}$ is generated in degrees at most $\operatorname{reg}\left(A^{\prime}\right)+1 \leq e(e-1)-2$. On the other hand, since $A^{\prime} \cong A / x B$, it follows that $\mathfrak{P}$ maps onto $x B$. Therefore the graded $A$-module $B \cong x B[e-1]$ is generated in degrees at most $e(e-1)-2-(e-1)=e(e-2)-1$.

The estimate for $\operatorname{embdim}(B)$ now follows from Proposition 6.1(b).

Remark 6.4. In Theorem 6.3 one can delete the assumption on $k$ and replace the condition $R_{2}$ by $R_{1}$ if one assumes that (6.2) holds in dimension $d-1$ over arbitrary fields. In this case one can pass to a purely transcendental field extension of $k$ and apply [14 Theorem] instead of [9, 4.10]. On the other hand, if (6.2) is only assumed for isolated singularities of dimension $d-1$, then the theorem holds with the additional assumption that $A$ is an isolated singularity. To see this notice that the property of being an isolated singularity passes from $A$ to the $\operatorname{ring} A^{\prime}$ in the proof of the theorem.

Theorem 6.5. Let $k$ be a perfect field, let $A$ be a reduced and equidimensional standard graded $k$-algebra of dimension $d$ and multiplicity $e \geq 2$, and let $A \subset B$ be a finite and birational extension of graded rings. If $A$ satisfies $R_{1}$ and $\operatorname{depth}_{A} B \geq$ $d-1$, then the graded $A$-module $B$ is generated in degrees at most $3 e-5$.

Proof. We may assume that $k$ is infinite and then by Proposition 5.5 we may reduce to the case where $A=k\left[x_{1}, \ldots, x_{d+2}\right]$ with the $x_{i}$ linear forms. Take $x_{1}, \ldots, x_{d+2}$ to be general, map the polynomial ring $R=k\left[X_{1}, \ldots, X_{d+2}\right]$ onto $A$ by sending $X_{i}$ to $x_{i}$, and write $T=k\left[X_{1}, \ldots, X_{d-1}\right] \subset R, S=k\left[x_{1}, \ldots, x_{d+1}\right] \subset A$. We may assume that the extension $k\left[x_{1}, \ldots, x_{d}\right] \subset A$ is finite, that the extensions $S \subset A$ 
and $k\left[x_{1}, \ldots, x_{d}, x_{d+2}\right] \subset A$ are finite and birational, and that $B / S$ and $S / J(S)$ are finite $T$-modules. One has $S=k\left[X_{1}, \ldots, X_{d+1}\right] /(f)$ for some form $f$ of degree $e$. Since $S / J(S)$ is a finite $T$-module, there exists a form $g \in k\left[X_{1}, \ldots, X_{d+1}\right]$ of degree $e-1$ so that $X_{1}, \ldots, X_{d-1}, f, g$ is a regular sequence on $k\left[X_{1}, \ldots, X_{d+1}\right]$ and the image of $g$ in $S$ lies in $J(S)$, hence in the conductor of $S$ (20. Theorem $2])$. Now write $k\left[x_{1}, \ldots, x_{d}, x_{d+2}\right]=k\left[X_{1}, \ldots, X_{d}, X_{d+2}\right] /(h)$ where $h$ is a form of degree $e$. As $h$ is monic in $X_{d+2}$, it follows that $X_{1}, \ldots, X_{d-1}, f, g, h$ is an $R$-regular sequence.

Consider the ring $A^{\prime}=R /(f, g, h)$. Since the socle of the complete intersection $A^{\prime} /\left(x_{1}, \ldots, x_{d-1}\right)$ is concentrated in degree $\operatorname{deg} f+\operatorname{deg} g+\operatorname{deg} h-3=3 e-4$, we conclude that the graded $T$-module $A^{\prime}$ is generated in degrees at most $3 e-4$. But $A^{\prime}$ maps onto $A / g A$, which in turn maps onto $A / S$ since $g$ is in the conductor of $S$. Thus as a $T$-module, $A / S$ is generated in degrees at most $3 e-4$.

Now consider the exact sequence of finite graded $T$-modules,

$$
0 \rightarrow A / S \longrightarrow B / S \longrightarrow B / A \rightarrow 0 \text {. }
$$

Here $B / S$ is a maximal Cohen-Macaulay module, hence free. Thus $A / S$ is a first syzygy module of $B / A$. But $A / S$ is generated in degrees at most $3 e-4$, and $B / A$ has no nontrivial free summand as $\operatorname{dim} B / A \leq d-2<\operatorname{dim} T$. It follows that the $T$-module $B / A$ is generated in degrees at most $3 e-4-1=3 e-5$, and then the same holds for the $A$-module $B$.

Theorem 6.6. Let $k$ be an algebraically closed field of characteristic zero and let $A$ be a standard graded domain over $k$ of dimension d and multiplicity $e \geq 2$. Let $A \subset B$ be a finite and birational extension of graded rings with $t=\operatorname{depth}_{A} B$.

(a) If $A$ satisfies $R_{d-t}$, then the graded $A$-module $B$ is generated in degrees at most $(d-t+1)(e-2)$, and

$\operatorname{embdim}(B) \leq\left(\begin{array}{c}(d-t+2)(e-1) \\ d-t+1\end{array}\right)-\left(\begin{array}{c}(d-t+1)(e-1)-1 \\ d-t+1\end{array}\right)+d$.

(b) If $t=d-1$ and $\operatorname{dim}_{A}(B / A) \leq d-2$, or if $t=d-2$ and $A$ satisfies $R_{2}$, or if $t=d-3$ and $A$ satisfies $R_{3}$, then the graded $A$-module $B$ is generated in degrees at most $e-3$, and

$$
\operatorname{embdim}(B) \leq\left(\begin{array}{c}
2 e+d-t-3 \\
d-t+1
\end{array}\right)-\left(\begin{array}{c}
e+d-t-3 \\
d-t+1
\end{array}\right)+d .
$$

Proof. By Theorem 2.1 we may assume that $B$ is not Cohen-Macaulay. Let $\mathbf{x}=$ $x_{1}, \ldots, x_{t-1}$ be a sequence of general linear forms in $A$, set $B^{\prime}=B /(\mathbf{x}) B$, and write $A^{\prime}$ for the image of $A$ in $B^{\prime}$. Our assumptions and conclusions are preserved by replacing $A \subset B$ with $A^{\prime} \subset B^{\prime}$, which can be seen as in the proof of Theorem 5.6. also notice that $B^{\prime}$ is a domain by $[9,4.10]$ since $d-(t-2) \geq 3$. Now we may assume that $d=d-t+1$, and hence $\operatorname{dim}_{A}(B / A)=0$.

Let $\mathfrak{m}$ denote the homogeneous maximal ideal of $A$. The exact sequence of $A$-modules

$$
0 \rightarrow A \longrightarrow B \longrightarrow B / A \rightarrow 0
$$

yields an embedding of local cohomology modules

$$
B / A=H_{\mathfrak{m}}^{0}(B / A) \hookrightarrow H_{\mathfrak{m}}^{1}(A) .
$$

Now in (a), $A$ is an isolated singularity and thus according to a result of Mumford ([3, 3.12.b], [5, 2.1]), $H_{\mathfrak{m}}^{1}(A)$ is concentrated in degrees at most $d(e-2)$. As $d(e-2) \geq$ 
0 , (5) then shows that the graded $A$-module $B$ is generated in degrees at most $d(e-2)$. As to (b), first notice that $\operatorname{ecodim}(A) \geq 2$ since otherwise $A$ would be Cohen-Macaulay and then $A=B$, forcing $B$ to be Cohen-Macaulay. Furthermore either $d=2$, or $d=3$ and $A$ is an isolated singularity, or $d=4$ and $A$ is an isolated singularity. Therefore $\operatorname{reg}(A) \leq e-\operatorname{ecodim}(A)$ by [12, 1.1], [19, Corollary] and [17, Theorem 2], respectively, except when $d=4$ and $\operatorname{ecodim}(A) \geq 3$ in which case $\operatorname{reg}(A) \leq e-\operatorname{ecodim}(A)+1$ according to [16, 3.1]. Under either assumption $\operatorname{reg}(A) \leq e-2$ and therefore $H_{\mathfrak{m}}^{1}(A)$ is concentrated in degrees at most $\operatorname{reg}(A)-1 \leq$ $e-3$. Finally $e-3 \geq 0$ since $e \geq \operatorname{ecodim}(A)+1$; see for instance [8, p. 112]. Now again (5) finishes the proof.

The assertions about $\operatorname{embdim}(B)$ are immediate consequences of these estimates and Proposition 6.1(b); if $e=3$ in part (b), one also uses the fact that ecodim $(A) \leq$ $e-1=2$.

Remark 6.7. For other values of $t=\operatorname{depth}_{A} B$ we obtain bounds that are only slighly weaker than the ones in Theorem 6.6(b). Indeed, if $t=d-4$ and $A$ satisfies $R_{4}$, then the graded $A$-module $B$ is generated in degrees at most $e+1$, as can be seen using [16, 3.2] in the proof of Theorem 6.6(b). If $t=d-5$ and $A$ satisfies $R_{5}$, then the graded $A$-module $B$ is generated in degrees at most $e+7$ by 18 , Theorem 3.1 and Proposition 3.1] and the proof of Theorem 6.6(b). Finally, if $t=d-6$ and $A$ satisfies $R_{6}$, then the graded $A$-module $B$ is generated in degrees at most $e+17$ according to [18, Theorem 3.1 and Proposition 3.2].

Corollary 6.8. Let $k$ be a field of characteristic zero and let $A$ be a reduced and equidimensional standard graded $k$-algebra of dimension $d \geq 2$ and multiplicity $e \geq 3$. If $A$ satisfies $R_{d-2}$, then for $B=\bar{A}$,

$$
\operatorname{embdeg}(B) \leq(d-1)(e-2) .
$$

We will now discuss degree bounds that could be derived from the complexity theory for Gröbner bases. Let $k$ be a field of characteristic zero, let $A$ be a reduced and equidimensional standard graded $k$-algebra of multiplicity $e \geq 2$, and let $A \subset B$ be a finite and birational extension of graded rings. Suppose that $A$ satisfies the condition $R_{1}$ and $B$ the condition $S_{2}$. Following the proof of Corollary 5.4 one can construct two forms $f, g$ of degree $e-1$ in the conductor of $A$ that generate an ideal of height 2. As the discussion of [27, Theorem 6.4.5] shows, one has

$$
B=A:(f, g) \text {, }
$$

where the colon is taken in the total ring of quotients of $A$. In other words, the elements of $B$ are obtained from the syzygies $a \cdot f-b \cdot g=0$ as they give rise to $b / f \in B$. In fact, collecting the coefficient ideal $L$ of $g$ in these syzygies, we obtain

$$
B=L f^{-1}=A\left[L f^{-1}\right] .
$$

This calculation is realized as follows. Write $A=R / I$, where $R$ is a polynomial ring in $n=\operatorname{embdim}(A)$ variables. For a set $f_{1}, \ldots, f_{m}$ of homogeneous generators of $I$, we consider the syzygies $\mathcal{S}$ of $f_{1}, \ldots, f_{m}, F, G$ over $R$, where $F$ and $G$ are homogeneous lifts of $f$ and $g$. The desired syzygies over $A$ are the images of $\mathcal{S}$ in A.

Let $\mathcal{G}=\left\{g_{1}, \ldots, g_{s}\right\}$ be a Gröbner basis of the ideal $J=\left(f_{1}, \ldots, f_{m}, F, G\right)$, and write $\delta=\max \left\{\operatorname{deg} f_{1}, \ldots, \operatorname{deg} f_{m}, e-1\right\}$. The set $\mathcal{S}$ will arise from division relations among the $g_{i}$ 's and therefore the coefficients of $\mathcal{S}$ will be generated in degrees at 
most $\Delta=\max \left\{\operatorname{deg} g_{i}\right\}$. According to [4, Appendix], and especially [11, Theorem $\mathrm{B}], \Delta$ is bounded by a polynomial in $\delta$ of degree $a^{n}$ (with $a$ in the order of $\sqrt{3}$ ). This is obviously much larger than the bounds of Theorems 6.3, 6.5 and 6.6.

\section{REFERENCES}

[1] M. Artin and M. Nagata, Residual intersections in Cohen-Macaulay rings, J. Math. Kyoto Univ. 12 (1972), 307-323. MR 46:166

[2] M. Auslander and D. Buchsbaum, On ramification theory in Noetherian rings, Amer. J. Math. 81 (1959), 749-765. MR21:5659

[3] D. Bayer and D. Mumford, What can be computed in algebraic geometry? Computational Algebraic Geometry and Commutative Algebra, Proceedings, Cortona 1991 (D. Eisenbud and L. Robbiano, Eds.), Cambridge University Press, Cambridge, 1993, 1-48. MR95d:13032

[4] T. Becker, H. Kredel and V. Weispfenning, Gröbner Bases, Springer, Heidelberg, 1993. MR $95 \mathrm{e}: 13018$

[5] A. Bertram, L. Ein and R. Lazarsfeld, Vanishing theorems, a theorem of Severi, and the equations defining projective varieties, J. Amer. Math. Soc. 4 (1991), 587-602. MR.92g:14014

[6] W. Bruns and J. Herzog, Cohen-Macaulay Rings, Cambridge University Press, Cambridge, 1993. MR $95 \mathrm{~h}: 13020$

[7] L. van den Dries and K. Schmidt, Bounds in the theory of polynomial rings over fields. A nonstandard approach, Invent. Math. 76 (1984), 77-91. MR85i:12016

[8] D. Eisenbud and S. Goto, Linear free resolutions and minimal multiplicity, J. Algebra $8 \mathbf{8}$ (1984), 89-133. MR85f:13023

[9] H. Flenner, Die Sätze von Bertini für lokale Ringe, Math. Ann. 229 (1977), 97-111. MR.57:311

[10] O. Forster, Über die Anzahl der Erzeugenden eines Ideals in einem Noetherschen Ring, Math. Z. 84 (1964), 80-87. MR29:1231

[11] M. Giusti, Some effectivity problems in polynomial ideal theory, EUROSAM 1984, Lecture Notes in Computer Science 174, Springer, Heidelberg, 1984, 159-171. MR86d:12001

[12] L. Gruson, R. Lazarsfeld and C. Peskine, On a theorem of Castelnuovo, and the equations defining space curves, Invent. Math. 72 (1983), 491-506. MR85g:14033

[13] M. Hochster, Rings of invariants of tori, Cohen-Macaulay rings generated by monomials, and polytopes, Ann. of Math. 96 (1972), 318-337. MR 46:3511

[14] M. Hochster, Properties of Noetherian rings stable under general grade reduction, Arch. Math. 24 (1973), 393-396. MR 48:8485

[15] T. de Jong, An algorithm for computing the integral closure, J. Symbolic Computation 26 (1998), 273-277. MR99d:13007

[16] S. Kwak, Castelnuovo regularity for smooth subvarieties of dimensions 3 and 4, J. Algebraic Geom. 7 (1998), 195-206. MR2000d:14043

[17] S. Kwak, Castelnuovo-Mumford regularity bound for smooth threefolds in $\mathbb{P}^{5}$ and extremal examples, J. reine angew. Math. 509 (1999), 21-34. MR.2000e:14064

[18] S. Kwak, Generic projections, the equations defining projective varieties and Castelnuovo regularity, Math. Z. 234 (2000), 413-434. MR2001e:14042

[19] R. Lazarsfeld, A sharp Castelnuovo bound for smooth surfaces, Duke Math. J. 55 (1987), 423-429. MR89d:14007

[20] J. Lipman and A. Sathaye, Jacobian ideals and a theorem of Briançon-Skoda, Michigan Math. J. 28 (1981), 199-222. MR83m:13001

[21] E. Noether, Idealdifferentiation und Differente, J. reine angew. Math. 188 (1950), 1-21. MR:12:388b

[22] A. Seidenberg, Construction of the integral closure of a finite integral domain II, Proc. Amer. Math. Soc. 52 (1975), 368-372. MR54:12741

[23] A. Simis, B. Ulrich and W. V. Vasconcelos, Tangent star cones, J. reine angew. Math. 483 (1997), 23-59. MR97m:14001

[24] A. Simis, B. Ulrich and W. V. Vasconcelos, Codimension, multiplicity and integral extensions, Math. Proc. Camb. Phil. Soc. 130 (2001), 237-257. MF.2002c:13017

[25] G. Stolzenberg, Constructive normalization of an algebraic variety, Bull. Amer. Math. Soc. 74 (1968), 595-599. MR 37:201 
[26] W. V. Vasconcelos, Computing the integral closure of an affine domain, Proc. Amer. Math. Soc. 113 (1991), 633-638. MR.92b:13013

[27] W. V. Vasconcelos, Computational Methods in Commutative Algebra and Algebraic Geometry, Springer, Heidelberg, 1998. MR99c:13048

Department of Mathematics, Purdue University, West Lafayette, Indiana 47907-1395

E-mail address: ulrich@math.purdue.edu

Department of Mathematics, Rutgers University, 110 Frelinghuysen Rd., Piscataway, NEW JERSEY 08854-8019

E-mail address: vasconce@math.rutgers.edu 